\title{
Ensayo clínico aleatorizado para evaluar dos pautas de administración del ibuprofeno en el tratamiento del ductus arterioso persistente eco-guiado.
}

\author{
María Carmen Bravo, Rebeca Sánchez ${ }^{1}$, Ana Isabel Blanco ${ }^{1}$, María Teresa Moral', Manuela López ${ }^{3}$, Fernando \\ Cabañas $^{3}$, Rocío Mosqueda², Izaskun Dorronsoro ${ }^{3}$, Laura Sánchez ${ }^{1}$, Paloma López ${ }^{1}$, Rosario Madero ${ }^{4}$, Adelina Pellicer ${ }^{1}$ \\ ${ }_{1}^{1}$ Departamento de Neonatología. Hospital Universitario La Paz, Madrid. España. \\ 2Departamento de Neonatología. Hospital Universitario Doce de Octubre, Madrid. España. \\ 'Departamento de Neonatología. Hospital Universitario Hospital Quirón \\ Salud Madrid y Quirón Salud San José, Madrid. España. \\ ${ }^{4}$ Departamento de Estadística. Hospital Universitario La Paz, Madrid. España. \\ Correspondencia: \\ María Carmen Bravo. Departamento de Neonatología. Hospital Universitario La Paz, Madrid. España. Paseo de la Castellana, 261. 28046. Madrid. \\ Email: mcarmen.bravo@salud.madrid.org. Tfno: +34917277416
}

\section{Summary:}

Patent ductus arteriosus (PDA) is a common condition of preterm infants and it is associated to significant morbidity and mortality. The best therapeutic option for PDA closure remains controversial. Necrotizing enterocolitis (NEC) and bowel spontaneous perforation have been observed in those infants who received pharmacologic treatment of PDA. Conventional treatment of PDA consists on 3 daily doses of intravenous (iv) ibuprofen in bolus $10-5-5 \mathrm{mg} / \mathrm{kg} / \mathrm{day}$. Recently, it has been shown that intravenous continuous infusion of ibuprofen (10-5-5 mg/kg/day) seems to be more efective for PDA closure than the convencional treatment with iv bolus. Our group demonstrated in a recent pilot study that the Echocardiographically guided trearment, EchoG, of PDA when it is compared with the convencional treatment, is feasible and reduces the number of ibuprofeno doses. Thus, EchoG treatment potentially reduces the adverse events associated to ibuprofen (i.e lower rate of NEC in the experimental group). This multicenter study will evaluate whether the combination of both experimental therapies (EchoG and iv continuous infusion) reduces the incidence of bowel adverse events (NEC and bowel spontaneous perforation) as compared with EchoG and iv ibuprofen bolus.

\section{Resumen:}

El ductus arterioso persistente (DA) es una entidad frecuente en el recién nacido prematuro y se asocia a morbilidad y mortalidad. Aún existe controversia sobre cuál es el mejor tratamiento para su cierre. Los niños con DA que reciben tratamiento farmacológico presentan con mayor frecuencia que el resto de niños prematuros,

enterocolitis necrotizante (ECN) o perforación intestinal aislada (PIA). En el momento actual, el tratamiento convencional del DA consiste en la administración de ibuprofeno (IB) intravenoso, iv, en bolo lento en 3 dosis diarias 10-5-5 mg/ $\mathrm{kg} /$ día. Recientemente, se ha observado que el tratamiento con IB en perfusión continua iv durante 3 días $10-5-5 \mathrm{mg} / \mathrm{kg} /$ día, parece ser más eficaz para cerrar el DA que el tratamiento convencional durante 3 días con la misma dosis pero en bolo iv lento. Dicho tratamiento experimental redujo la incidencia de ECN asociada. Nuestro grupo demostró en un ensayo piloto previo que el tratamiento guiado con ecocardiografía, EchoG del DA con IB iv comparado con el tratamiento convencional, permite disminuir el número de dosis al

paciente. El tratamiento EchoG presenta así una potencial reducción de los efectos secundarios asociados a la medicación, esto se tradujo en una tendencia a presentar menor incidencia de ECN en el grupo experimental. Con este ensayo clínico multicéntrico se pretende testar la hipótesis de que la combinación de 2 tratamientos experimentales, la utilización de IB en perfusión continua y EchoG, reduce la incidencia de efectos secundarios digestivos frente al tratamiento también guiado por ecocardiografía pero en bolo lento iv.

Keywords: Ductus arteriosus, ibuprofen, necrotizing enterocolitis, echocardiography. / Ductus arterioso, ecocardiografía, ibuprofeno, enterocolitis necrotizante.

Recibido 22 de octubre, 2018 Aceptado 21 de febrero, 2019 Publicado 5 de junio, 2019.

Copyright: (c) 2019 Authors. This is an open-access article distributed under the terms of the Creative Commons Attribution License, which permits unrestricted use, distribution, and reproduction in any medium, provided the original author and source are credited. Editor: Alberto Borobia

Cite as: Bravo MC., Sánchez R., Blanco Al., Moral MT., López M, Cabañas F., Mosqueda R., Dorronsoro I., Sánchez L., López P., Madero R., Pellicer A. Ensayo clínico aleatorizado para evaluar dos pautas de administración del ibuprofeno en el tratamiento del ductus arterioso persistente eco-guiado. IBJ Clin Pharmacol 2019 1(1):e0012. DOI: 10.24217/2530-4984.19v1s1.00012 Funding: PI16/00644: Convocatoria AES 2016, regulada mediante la Resolución del Instituto de Salud Carlos III. XXIII Ayuda a la Investigación de la MM. Código del protocolo: IBU24h-EchoG / Código EudraCT: 2016-002974-11

Competing Interests: The authors declare no conflicts of interest. 


\section{INTRODUCCIÓN}

El ductus arterioso persistente (DA) aunque es una enfermedad rara a nivel poblacional $(<5: 10.000$ habitantes) es una entidad que se asocia a una alta morbilidad y mortalidad. Aún existe controversia sobre cuál es el mejor tratamiento para su cierre. Los niños con DA que reciben tratamiento farmacológico presentan con mayor frecuencia que el resto de niños prematuros, enterocolitis necrotizante (ECN) o perforación intestinal aislada (PIA), sin que se sepa si esta asociación se debe al DA en sí mismo, a los fármacos que se utilizan para su cierre o a la propia inmadurez de los pacientes. En el momento actual, el tratamiento convencional del DA consiste en la administración de un ciclo de ibuprofeno (IB) intravenoso (iv), que consiste en la administración en bolo lento de 1 dosis diaria del fármaco con una pauta de dosificación 10-5-5 mg/kg/día durante 3 días consecutivos. En el caso en que el DA no se cierre con el primer ciclo de IB, puede utilizarse un segundo ciclo del fármaco siguiendo el mismo esquema. Existen 2 aspectos claves que podrían mejorar el cuidado de los niños prematuros que reciben tratamiento médico para el cierre del DA: incrementar la eficacia terapéutica de los tratamientos utilizados y reducir los efectos secundarios asociados a la medicación.

Se ha ensayado recientemente el tratamiento con IB iv en perfusión continua durante 3 días con una dosis inicial de $10 \mathrm{mg} / \mathrm{kg} /$ día seguida de $5 \mathrm{mg} / \mathrm{kg} /$ día en las $48 \mathrm{~h}$ siguientes (dosis total acumulada $20 \mathrm{mg} / \mathrm{kg}$ ). Este tratamiento experimental fue más eficaz para cerrar el DA que el tratamiento convencional con la misma dosis pero en bolo (administración iv lenta en $15 \mathrm{~min}$ ) en un ensayo clínico realizado sobre 111 niños prematuros (1). Sorprendentemente, el IB en perfusión continua fue más eficaz en la población más inmadura, que es aquella con mayor tasa de fracaso terapéutico y más vulnerable a los efectos secundarios de la medicación.

Hasta la fecha no ha sido esclarecido el efecto que el DA o su tratamiento ejerce en el territorio esplácnico. La ECN es una enfermedad devastadora en la población de niños prematuros y se asocia a mortalidad, displasia broncopulmonar, retinopatía de la prematuridad, leucomalacia periventricular e infección nosocomial (3). Existen estudios epidemiológicos que sugieren una relación entre el DA y la ECN (4). Se ha observado que existe una disminución del flujo sanguíneo en el tronco celíaco a partir del tercer día de vida en presencia de DA (5). Existe discusión sobre la asociación de la ECN o PIA y los fármacos que se utilizan para cerrar el DA. La tasa global de ECN en los pacientes prematuros se encuentra entre el 4-12\%, siendo esta incidencia del 8-12\% en el grupo de recién nacidos prematuros que presentan DA y que reciben IB para su cierre $(1,6)$. El único ensayo clínico realizado hasta la fecha con IB en perfusión iv continua consiguió una reducción en la incidencia de ECN que rozó el límite de la significación estadística, cuando se comparó con el tratamiento convencional $(8,9 \%$ vs $0 \%)$ $(\mathrm{p}=0,057)(1)$.
También se ha intentado reducir la probabilidad de efectos secundarios asociados al uso de IB realizando tratamiento ecocardiográficamente guiado (EchoG) del ductus, consistente en la administración de una primera dosis del fármaco, siendo indicadas las dosis sucesivas en función de los hallazgos ecocardiográficos. Este tratamiento experimental, comparado con el convencional, demostró que el número de dosis recibida fue significativamente inferior (6). Además, el tratamiento experimental EchoG presentó menor incidencia de ECN ( $2 \%$ en grupo EchoG y $19 \%$ en tratamiento convencional, $p=0,3)(6)$. La tercera estrategia que podría reducir los efectos secundarios potencialmente asociados a la medicación utilizada para cerrar el DA es la identificación precoz de individuos genéticamente predispuestos al fracaso terapéutico, o aquéllos más vulnerables a desarrollar efectos adversos. En este sentido se han identificado polimorfismos genéticos en niños en los que el tratamiento médico fracasó y en los que fue necesario el cierre quirúrgico (7). También se han identificado polimorfismos relacionados con la ECN (8). Otros polimorfismos involucrados en el metabolismo del ibuprofeno también podría influir en el perfil de seguridad de este fármaco (9). La identificación precoz de niños genéticamente predispuestos al fracaso del tratamiento médico o al desarrollo de ECN asociada al tratamiento del DA sería extremadamente útil, ya que en ellos, probablemente, el tratamiento quirúrgico sería la primera opción terapéutica.

Este ensayo clínico pretende evaluar si el tratamiento ecocardiográficamente guiado del ductus arterioso persistente con IB en perfusión iv continua es capaz de reducir la incidencia de ECN o PIA al compararlo con el tratamiento ecocardiográficamente guiado con bolos iv lento.

\section{MATERIALES Y MÉTODOS}

Estudio aleatorizado y ciego, multicéntrico nacional, anidado en la red de Salud Materno-Infantil y Desarrollo (red SAMID). La inclusión de pacientes se realizará en los hospitales: Hospital La Paz de Madrid, Hospital Quirón Salud Madrid y Hospital Doce de Octubre. El ensayo clínico que se detalla a continuación se ajustará a la normativa vigente en relación a los ensayos clínicos con medicamentos de uso humano, (Real Decreto 1090/2015, de 4 de diciembre, por el que se regulan los ensayos clínicos con medicamentos, los Comités de Ética de la Investigación con medicamentos y el Registro Español de Estudios Clínicos).

\section{DISCUSIÓN}

El tratamiento del ductus arterioso es controvertido y se siguen buscando alternativas terapéuticas con mejor perfil de eficacia y seguridad que el tratamiento convencional. Con este ensayo clínico se pretende, en primer lugar, reducir la incidencia de ECN y PIA que se asocian a mortalidad y retraso en el desarrollo, limitándose la tasa de intervenciones abdominales por estas indicaciones. 
En segundo lugar, también se pretende aumentar la eficacia del IB para el cierre del DA. Esto tendrá como consecuencia inmediata la disminución del número de cirugías para el cierre del DA en los recién nacidos prematuros. La intervención quirúrgica de estos pacientes está asociada a mayor tasa de mortalidad y morbilidad tales como enfermedad pulmonar crónica, retinopatía de la prematuridad y retraso en el neurodesarrollo. Por último, la identificación precoz de individuos genéticamente predispuestos al fracaso terapéutico con IB o susceptibles a presentar con mayor frecuencia efectos secundarios graves, contribuiría a individualizar el tratamiento del DA y poder seleccionar pacientes candidatos a cierre quirúrgico como primera opción terapéutica.

\section{PACIENTES}

Los recién nacidos prematuros con menos 33 semanas de edad gestacional y $\mathrm{DA} \geq 1,5 \mathrm{~mm}$ con decisión de iniciar tratamiento farmacológico cuyos representantes legales firmen el consentimiento informado serán aleatorizados a recibir IB en perfusión iv continua y EchoG (grupo A) o IB en bolo iv lento y EchoG (grupo B), siempre que no presenten alteraciones congénitas graves, cardiopatías congénitas o contraindicación para la administración de IB [oligoanuria, sangrado intraventricular reciente grave (HIV grado III o infarto hemorrágico periventricular extenso), creatinina sérica $>1,5 \mathrm{mg} / \mathrm{dl}$ o sospecha clínica de isquemia intestinal].

Se utilizará el preparado comercial Pedea ${ }^{\circledR}$ (OrphanEurope, Paris, France), 5mg/ ml.

En el momento de la inclusión de los pacientes, se recogerá una muestra de DNA desde un frotis de epitelio yugal para estudio de polimorfismos: TFAP2B (rs987237) y EPAS1 (rs1867785), que son genes que se expresan en el músculo liso del DA y juegan un papel crucial en el cierre del mismo en modelos animales. Ambos genes participan en el proceso de transcripción que regula el desarrollo del músculo liso en el tejido ductal (7).

También se estudiarán polimorfismos relacionados con la ECN: el C-1625 G en la región promotora del gen MD-2, que se ha asociado con ECN graves; y el polimorfismo en la región codificante del gen GM2A, rs1048719 (G>A), en el exón 1 (8). Por último, se estudiarán polimorfismos involucrados en el metabolismo del ibuprofeno que podría influir en el perfil de seguridad de este fármaco (CYP2C9, el CYP2C8, CYP3A4 y CYP2C19) (9).

\section{PROCEDIMIENTOS DEL ESTUDIO}

Grupo A: Administración de IB EchoG en perfusión iv continua con una pauta de dosificación $10-5-5 \mathrm{mg} / \mathrm{kg} /$ día.

Grupo B: Administración de IB EchoG en bolo lento iv con una pauta de dosificación $10-5-5 \mathrm{mg} / \mathrm{kg} /$ día.

En ambos grupos de estudio, el tratamiento con ibuprofeno se realizará EchoG $(10,11)$, lo que significa que una vez administrada la primera dosis del fármaco, se evaluará con ecocardiografía la persistencia del ductus antes de administrar las dosis sucesivas. De esta forma, en aquellos casos en los que no se observe flujo ductal con ecocardiografía, no se administrarán las siguientes dosis de ibuprofeno.

Evaluaciones basales en el momento de la inclusión

- Análisis de polimorfismos genéticos.

Evaluaciones de seguimiento

- Cumplimiento de los procedimientos especificados para cada visita (ver tabla 7, Tabla de procedimientos).

\section{Ecocardiogramas}

Se harán todas las correspondientes por indicación clínica y además se realizarán:

- La primera ecocardiografía (previo al tratamiento) será realizada según práctica clínica habitual, siempre a cargo de un cardiólogo para descartar anomalías estructurales del corazón.

- Ecocardiografías diarias durante la fase de tratamiento (independientemente del número de ciclos), serán realizadas por los médicos tratantes

(investigadores).

- Ecocardiografías de control tras la finalización del ciclo de tratamiento: se reevaluará y se decidirá la necesidad de continuar con el tratamiento (nuevo ciclo) 24h después de la última dosis. La confirmación del cierre se realizará según práctica clínica habitual por un cardiólogo.

\section{Estudio genético}

Una vez aceptado el consentimiento informado por los padres o tutores se recogerá con dos hisopos dos muestras de la mucosa bucal. Se utilizará un kit comercial para tal fin. Se enviarán los hisopos al Instituto de Genética Médica y Molecular (INGEMM) en el Hospital Universitario La Paz donde se procederá a hacer la extracción del ADN. Se guardarán dos alícuotas. Una se destinará al Instituto de Nutrición y Tecnología de los

Alimentos "José Mataix" para determinación de polimorfismos genéticos mediante TaqMan ${ }^{\circledR}$ Probe. Se analizarán los polimorfismos TFAP2B (rs987237) y EPAS1 (rs1867785) y el C-1625 G en la región promotora del gen MD-2, así como el gen GM2A, rs1048719 (G>A) en el exón 1. Y otra alícuota de ADN se mantendrá en el INGEMM para la determinación de los polimorfismos del CYP2C9 (alelos 2 y 3), CYP2C8 (alelos 2 y 3) y los UGTs $1^{\mathrm{a}}$ y $2 \mathrm{~B}$, por tecnología OpenArray (ClinPharmArray $\left.{ }^{\circledR}\right)$.

Además, se cumplimentará el apartado correspondiente del CRD en cada etapa del estudio.

\section{$\underline{\text { Analíticas }}$}

Las analíticas tanto basales como de control, se harán según protocolo de cada Unidad de Neonatos y particularidades de cada paciente. Las analíticas para evaluar función renal a través de la creatinina y las gasometrías son de petición rutinaria en pacientes neonatos. 


\section{VARIABLES DE ESTUDIO}

\section{VARIABLE PRINCIPAL}

Incidencia de ECN o PIA, definida como la presencia de neumatosis intestinal, neumoperitoneo, o aire en vena porta.

\section{VARIABLES SECUNDARIAS}

Variables perinatales:

- Edad de la madre

- Edad gestacional

- Medicación materna (antibióticos, anticonvulsivantes, antihipertensivos, drogas de abuso, heparina, tocolíticos, etc).

- Peso al nacer

- Sexo

- Peso $(<\mathrm{P} 10)$

- Corticoides prenatales (ciclo completo)

- Apgar 5 minutos

- Amniorrexis $>18$ horas

- Reanimación avanzada al nacer

- SNAPPE II

- Corioamnionitis clínica

- Corioamnionitis confirmada histológicamente

- $\mathrm{pH}$ del cordón

- Embarazo múltiple

- Necesidad de surfactante

- Edad de inicio de tratamiento

Variables ecocardiográficas:

Previo al tratamiento:

- Tamaño de ductus con Doppler color en un corte supraesternal sagital.

- Relación AI/Ao (aurícula izquierda/aorta) en un corte paraesternal eje largo en modo $\mathrm{M}$.

- Velocidad diastólica en la rama pulmonar izquierda con un corte paraesternal sagital.

- Relación E/A (onda de llenado ventricular izquierdo) con una ventana apical 4 cámaras.

- FVCS (flujo en vena cava superior) midiendo el tamaño desde una ventana paraesternal izquierda media y la velocidad desde una ventana subcostal.

- Velocidad diastólica en aorta postductal desde un corte supraesternal.

Variables cardiorrespiratorias:

Previo a cada una de las dosis:

- Frecuencia cardiaca

- Presión arterial

- Frecuencia respiratoria

- Modalidad de asistencia respiratoria
- PMVA (presión media en vía aérea)

- $\mathrm{FiO} 2$

- $\mathrm{SatO} 2$

- pCO2 capilar

- pCO2 arterial

- pCO2 venosa

- EB (Exceso de bases)

- Ácido láctico

- Diuresis 24 horas previas al tratamiento

- Relleno capilar

- Gradiente de $\mathrm{T}^{\mathrm{a}}$ central-periférico

- Soporte cardiovascular previo al tratamiento (expansores de volemia en 24 horas previas, el score inotrópico previo a cada dosis de tratamiento $=($ dopamina $\mathrm{x} 1)+($ dobutamina $\mathrm{x} 1)+($ adrenalina $\mathrm{x} 10)$ o necesidad de hidrocortisona.

\section{Variables de evaluación de Eficacia:}

- Fracaso terapéutico, definido como la presencia de $\mathrm{DA} \geq 1,5 \mathrm{~mm} 24-48$ horas después de finalizar hasta un máximo de 6 dosis de ibuprofeno (2 ciclos completos).

- Número de dosis de ibuprofeno administrados.

- Tasa de cierre tras un ciclo de tratamiento

- Tasa de cierre tras dos ciclos de tratamiento.

- Tiempo necesario hasta el cierre.

- Necesidad de ligadura quirúrgica.

- Tasa de reapertura: definido como la presencia de flujo ductal por Doppler color tras haber confirmado cierre previo ecocardiográfico.

\section{Variables Genéticas:}

- Estudio de polimorfismos genéticos asociados a fracaso terapéutico o riesgo de enterocolitis necrotizante o perforación intestinal aislada.

- Estudio de polimorfismos genéticos asociados al metabolismo y eliminación del ibuprofeno y su relación con la aparición de acontecimientos adversos.

\section{Variables de Seguridad:}

- Mortalidad antes del alta de la unidad de neonatología

- Días de ingreso

- Enfermedad Pulmonar Crónica (EPC), definida como la necesidad de oxígeno suplementario a las 36 semanas de edad postmenstrual.

- Hemorragia pulmonar, diagnosticada ante la presencia de sangre fresca o secreción teñida de sangre extraída del tubo endotraqueal, asociado a deterioro respiratorio y radiológico o diagnóstico en autopsia.

- Hipertensión pulmonar secundaria, en caso de que el paciente presentara una presión pulmonar estimada por ecocariografía $\geq 60 \%$ de la presión sistémica, una vez iniciado el tratamiento con ibuprofeno asociado a deterioro respiratorio. 
- Tiempo en alcanzar la nutrición enteral exclusiva.

- Sangrado gastrointestinal, en caso de presencia de líquido sanguinolento o sangre fresca en los restos gástricos recogidos por aspiración de la sonda nasogástrica durante el tratamiento con ibuprofeno.

- Oligoanuria, definida como diuresis $<1 \mathrm{cc} / \mathrm{kg} / \mathrm{h}$.

- Diagnóstico de lesión estructural del sistema nervioso central por USC a la edad del término.

- Fármacos que el paciente ha recibido antes y durante el tratamiento con ibuprofeno.

\section{Estudio genético:}

- Estudio de polimorfismos genéticos asociados a fracaso terapéutico o riesgo de enterocolitis necrotizante o perforación intestinal aislada.

- Estudio de polimorfismos genéticos asociados al metabolismo y eliminación del ibuprofeno y su relación con la aparición de acontecimientos adversos.

\section{ALEATORIZACIÓN Y ENMASCARAMIENTO}

Se realizará una lista de asignación aleatoria de los pacientes para evitar sesgos de asignación, para aumentar la probabilidad de que las características conocidas y desconocidas de los pacientes (características demográficas y basales, entre otras) se distribuyan de forma equilibrada entre los dos grupos de estudio, así como para aumentar la validez de las comparaciones estadísticas entre los grupos de tratamiento. Una vez se obtenga el consentimiento, los pacientes serán asignados a cada grupo de tratamiento mediante un sistema de números aleatorios, según el programa de aleatorización preparado por la Unidad de Bioestadística del Hospital La Paz. Se utilizará una aleatorización estratificada por edad gestacional ( $<28$ semanas $\mathrm{y} \geq 28$ semanas) y centro hospitalario.

En cada centro hospitalario y en cada estrato se asignarán aleatoriamente los pacientes en una proporción 1:1 a cada grupo de estudio.

Una enfermera de neonatología que participará en el ensayo clínico pero que no se encargará del cuidado del recién nacido, preparará la medicación del estudio, de acuerdo a un código preestablecido con un aspecto externo indistinguible para el IB y el suero glucosado al $5 \%$. Para garantizar el ciego, los pacientes asignados al grupo A recibirán un bolo iv de suero glucosado al 5\% en 15 min que irá seguido de la infusión continua de IB a 0,1 $\mathrm{cc} / \mathrm{h}$ y los pacientes del grupo B recibirán el bolo iv de IB en 15 min que irá seguido de la infusión continua de suero glucosado al $5 \%$ a $0,1 \mathrm{cc} / \mathrm{h}$.

Tabla 1. Calendario y programación de procedimientos

\begin{tabular}{|c|c|c|c|c|c|c|c|c|}
\hline Actividades & $\begin{array}{l}\text { V1 } \\
\text { Día } 0\end{array}$ & v2 & v3 & v4 & v5 & v6 & $\begin{array}{c}\mathrm{V7} \\
\text { Fin de } \\
\text { Tratamiento }\end{array}$ & $\begin{array}{l}\text { Follow-up (último día de seguimiento) } \\
\text { Desde la última dosis al alta, fallecimeinto o } \\
40 \text { semanas de edad postmentrual. }\end{array}$ \\
\hline $\begin{array}{l}\text { Información al representate/tutor y Consentimiento } \\
\text { informado }\end{array}$ & $\mathrm{x}$ & & & & & & & \\
\hline Revisión criterios inclusión/exclusión & $\mathrm{x}$ & & & & & & & \\
\hline $\begin{array}{l}\text { Historia clínica/antecedentes personales, recoger } \\
\text { variables perinatales y medicación concomitante }\end{array}$ & $\mathrm{x}$ & & & & & & & \\
\hline Exploración Fisica & $\mathrm{x}$ & $\mathrm{x}$ & $\mathrm{x}$ & $\mathrm{x}$ & $\mathrm{x}$ & $\mathrm{x}$ & $\mathrm{x}$ & $\mathrm{x}$ \\
\hline Signos Vitales $^{a}$ & $x$ & $x$ & $\mathrm{x}$ & $x$ & $\mathrm{x}$ & $\mathrm{x}$ & $x$ & $x$ \\
\hline $\begin{array}{l}\text { Ecocardiografia. Recoger variables ecocardiográficas } \\
\text { iniciales }\end{array}$ & $\mathrm{x}$ & & & & & & & \\
\hline $\begin{array}{l}\text { Ecocardiografia para guiar el tratamiento y de } \\
\text { seguimiento }\end{array}$ & & $\mathrm{x}$ & $\mathrm{x}$ & $\mathrm{x}$ & $\mathrm{x}$ & $\mathrm{x}$ & $\mathrm{x}$ & $x^{b}$ \\
\hline Tratamiento (1er. Ciclo) ${ }^{\mathrm{C}}$ & $\mathrm{x}$ & $x$ & $\mathrm{x}$ & & & & & \\
\hline Tratamiento (2do. Ciclo) & $\mathrm{x}$ & & & $\mathrm{x}$ & $\mathrm{x}$ & $\mathrm{x}$ & & \\
\hline Extracción muestra para genotipo & $\mathrm{x}$ & & & & & & & \\
\hline Evaluación de parámetros de seguridad & $\mathrm{x}$ & $\mathrm{x}$ & $\mathrm{x}$ & $\mathrm{x}$ & $\mathrm{x}$ & $\mathrm{x}$ & $\mathrm{x}$ & $x$ \\
\hline $\begin{array}{l}\text { Evaluación variables cardiorespiratorias (previo a } \\
\text { cada dosis) }\end{array}$ & $\mathrm{x}$ & $\mathrm{x}$ & $\mathrm{x}$ & $\mathrm{x}$ & $\mathrm{x}$ & $\mathrm{x}$ & & \\
\hline Cuantificación de la diuresis & $\mathrm{x}$ & $\mathrm{x}$ & $\mathrm{x}$ & $\mathrm{x}$ & $\mathrm{x}$ & $\mathrm{x}$ & $\mathrm{x}$ & $\mathrm{x}$ \\
\hline Evaluación eficacia terapéutica & & $\mathrm{x}$ & $\mathrm{x}$ & $\mathrm{x}$ & $\mathrm{x}$ & $\mathrm{x}$ & $\mathrm{x}$ & $\mathrm{x}$ \\
\hline Recogida de Acontecimientos Adversos & $\mathrm{x}$ & $\mathrm{x}$ & $\mathrm{x}$ & $\mathrm{x}$ & $\mathrm{x}$ & $\mathrm{x}$ & $\mathrm{x}$ & $\mathrm{x}$ \\
\hline
\end{tabular}

a. Dentro del período de tratamiento, se medirá previo a la administración de cada dosis de ibuprofeno.

b. Ecocardiografía opcional si el DA quedó abierto en la visita 7.

c. Según criterio del médico responsable del paciente, en función de la información clínica y ecocardiogáfica que presente al finalizar la primera tanda. 


\section{DOSIS ADICIONALES DE IB}

Se evaluará con ecocardiografía la necesidad de administrar una segunda tanda de tratamiento con IB tras completar el ciclo de tres dosis en cada uno de los grupos. La decisión de iniciar una segunda tanda de tratamiento de 3 dosis, será decidido por el médico responsable del paciente en función de la información clínica y ecocardiográfica que presente al finalizar la primera tanda de tratamiento. En caso de decidir iniciar un nuevo ciclo terapéutico, seguirá el mismo esquema terapéutico al que fue aleatorizado (intervención A o B). El médico responsable del cuidado del recién nacido será quien decida si existe indicación de cirugía en caso de fracaso del tratamiento médico del DA.

El manejo del paciente se realizará según el protocolo vigente en la respectiva unidad a la que pertenezca. Se recomienda la restricción hídrica y evitar el uso de furosemida.

\section{TAMAÑO MUESTRAL}

La incidencia en el Hospital La Paz de ECN o perforación intestinal aislada en los recién nacidos que habían recibido IB entre 2010 y 2015 osciló entre un 8-9\% según el año de estudio. Por tanto, para reducir la incidencia del $9 \%$ al $0,1 \%$ con un poder estadístico del $80 \%$ y una $\mathrm{p}<0,05$, sería necesario incluir 180 pacientes, 90 niños por grupo de estudio. El período de reclutamiento tendrá una duración de 24 meses.

\section{ANÁLISIS ESTADÍSTICO}

Los datos se analizarán con el paquete estadístico SPSS 25.0 ${ }^{\circledR}$ y SAS 9.1.3 ${ }^{\circledR}$ para Windows (Cary, NC, USA).

Se diseñará una base de datos que refleje exactamente el contenido del cuaderno de recogida de datos (CRD), en la que se establezca la matriz de entrada de datos con los rangos o valores posibles, así como las diferentes reglas de coherencia entre variables. Se controlará la calidad de la información recibida mediante la realización de un análisis exploratorio orientado a la detección de valores discrepantes, fuera de rango o ausentes. El análisis exploratorio también informará respecto a la distribución de las principales variables a estudiar y orientará sobre posibles transformaciones. El análisis de datos se realizará basándose en intención de tratamiento. Se presentarán estadísticas descriptivas resumen de las variables cuantitativas incluyendo: número de niños en el grupo, media (m), desviación típica (DE), mediana (M), mínimo, máximo y cuartiles 25\% (Q25) y 75\% (Q75). Para los datos categóricos, se presentarán las distribuciones de frecuencia (absoluta y relativa). Las características demográficas y antecedentes se resumirán por grupo de estudio para evaluar la comparabilidad de los grupos de tratamiento en el momento basal. Las comparaciones entre variables cuantitativas continuas se realizarán principalmente mediante pruebas paramétricas, utilizando la $t$ de Student. En caso de tamaño muestral menor de
30 individuos, la significación estadística en este tipo de análisis se obtendrán mediante pruebas no paramétricas, Kruskal-Wallis o U de Mann-Whitney. El análisis de frecuencias entre variables cualitativas se realizará mediante la prueba de la Chi cuadrado o el test exacto de Fisher, en caso de tamaño muestral inferior a 20. Cuando se utilice la Chi cuadrado se aplicará en todos los casos la corrección de Yates. Todas las pruebas serán consideradas bilaterales y se definirán valores estadísticamente significativos si $p<0,05$. Se realizará un análisis intermedio sin abrir el ciego tras 1 año de reclutamiento para analizar si existen diferencias significativas entre ambos grupos de estudio.

\section{DISCUSIón}

El tratamiento del ductus arterioso es controvertido y se siguen buscando alternativas terapéuticas con mejor perfil de eficacia y seguridad que el tratamiento convencional. Con este ensayo clínico se pretende, en primer lugar, reducir la incidencia de ECN y PIA que se asocian a mortalidad y retraso en el desarrollo, limitándose la tasa de intervenciones abdominales por estas indicaciones. En segundo lugar, también se pretende aumentar la eficacia del IB para el cierre del DA. Esto tendrá como consecuencia inmediata la disminución del número de cirugías para el cierre del DA en los recién nacidos prematuros. La intervención quirúrgica de estos pacientes está asociada a mayor tasa de mortalidad y morbilidad tales como enfermedad pulmonar crónica, retinopatía de la prematuridad y retraso en el neurodesarrollo. Por último, la identificación precoz de individuos genéticamente predispuestos al fracaso terapéutico con IB o susceptibles a presentar con mayor frecuencia efectos secundarios graves, contribuiría a individualizar el tratamiento del DA y poder seleccionar pacientes candidatos a cierre quirúrgico como primera opción terapéutica.

\section{AGRADECIMIENTO}

Los autores agradecen a la red SAMID (RD12/0026/004) su consejo científico. Este trabajo ha contado con el apoyo de la Plataforma Española de Investigación Clínica y Ensayos Clínicos, SCReN (Spanish Clinical Research Network), financiada por el ISCIIISubdirección General de Evaluación y Fomento de la Investigación, mediante el proyecto PT13/0002/0015 y el proyecto PT17/0017/0013 integrado en el Plan Estatal de I+D+I 2013-2016 y por el Plan Estatal de Investigación Científica y Técnica y de Innovación (2017-2020) y cofinanciado por el Fondo Europeo de Desarrollo Regional (FEDER) "Una manera de hacer Europa". 


\section{INVESTIGADORES}

\section{INVESTIGADOR COORDINADOR}

Dra. María Carmen Bravo Laguna

Servicio de Neonatología

Hospital Universitario La Paz

Paseo de la Castellana, 261

Madrid - 28046

e-mail: mcarmen.bravo@salud.madrid.org

\section{INVESTIGADORES COLABORADORES}

Dra. Adelina Pellicer

Servicio de Neonatología. Hospital

Universitario La Paz. Madrid.

Dra. Marta Ybarra

Servicio de Neonatología. Hospital

Universitario La Paz. Madrid.

Dra. Paloma López

Servicio de Neonatología. Hospital

Universitario La Paz. Madrid.

Dra. Laura Sánchez

Servicio de Neonatología. Hospital

Universitario La Paz. Madrid.

Dra Rebeca Sánchez

Servicio de Neonatología. Hospital

Universitario La Paz. Madrid.

Dra Ana Isabel Blanco

Servicio de Neonatología. Hospital

Universitario La Paz. Madrid.

Dr. Fernando Cabañas*

Servicio de Neonatología. Hospital Quirón

Salud Madrid y Quirón Salud San José

Dra. Manuela López Azorín Servicio de Neonatología. Hospital Quirón

Salud Madrid y Quirón Salud San José

Dr. Izaskun Dorronsoro

Servicio de Neonatología. Hospital Quirón

Salud Madrid y Quirón Salud San José

Dra. María Teresa Moral**

Servicio de Neonatología. Hospital

Universitario Doce de Octubre. Madrid

Dra. Rocío Mosqueda

Servicio de Neonatología. Hospital

Universitario Doce de Octubre. Madrid

* IP del Hospital Quirón Salud Madrid y Quirón Salud San José

** IP del Hospital Universitario Doce de Octubre

\section{BIBLIOGRAFÍA}

[1]. Lago P, Salvadori S, Opocher F, Ricato S, Chiandetti L, Frigo AC. Continuous infusion of ibuprofen for treatment of patent ductus arteriosus in very low birth weight infants.Neonatology. 2014;105(1):46-54

[2]. Noori S, McCoy M, Friedlich P, Bright B, Gottipati $\mathrm{V}$, Seri I, et al. Failure of ductus arteriosus closure is associated with increased mortality in preterm infants. Pediatrics 2009 Jan;123(1):e138-44.

[3]. Shah J, Singhal N, da Silva O, Rouvinez-Bouali N, Seshia M, Lee SK, Shah PS; Canadian Neonatal Network. Intestinal perforation in very preterm neonates: risk factors and outcomes. J Perinatol. 2015 Aug;35(8):595-600.

[4]. Grosfeld JL, ChAAt M, Molinari F, Engle W, Engum SA, West KW, et al. Increased risk of necrotizing enterocolitis in premature infants with patent ductus arteriosus treated with indomethacin. Ann Surg 1996 Sep;224(3):350-5; discussion 355-7.

[5]. El-Khuffash A, Higgins M, Walsh K, Molloy EJ. Quantitative assessment of the degree of ductal steal using celiac artery blood flow to left ventricular output ratio in preterm infants. Neonatology 2008;93(3):206212.

[6]. Bravo MC, Cabañas F, Riera J, Pérez-Fernández E, Quero J, Pérez-Rodríguez J, Pellicer A.

Randomised controlled clinical trial of standard versus echocardiographically guided ibuprofen treatment for patent ductus arteriosus in preterm infants: a pilot study. J Matern Fetal Neonatal Med. 2014 Jun;27(9):904-9.

[7]. Dagle JM, Lepp NT, Cooper ME, Schaa KL, Kelsey KJ, Orr KL, Caprau D, Zimmerman CR, Steffen KM, Johnson KJ, Marazita ML, Murray JC. Determination of genetic predisposition to patent ductus arteriosus in preterm infants. Pediatrics. 2009 Apr;123(4):1116-23

[8]. Zhou W, Yuan W, Huang L, Wang P, Rong X, Tang J. Association of neonatal necrotizing enterocolitis with myeloid differentiation-2 and GM2 activator protein genetic polymorphisms.Mol Med Rep. 2015 Jul;12 (1):974-80

[9]. Mazaleuskaya LL, Theken KN, Gong L, Thorn CF, FitzGerald GA, Altman RB, Klein TE. PharmGKB summary: ibuprofen pathways. Pharmacogenet Genomics. 2015 Feb;25(2):96-106.

[10]. Carmo KB, Evans N, Paradisis M. Duration of indomethacin treatment of the preterm patent ductus arteriosus as directed by echocardiography. J Pediatr 2009;155:819 -822. 10.

[11]. Su BH, Peng CT, Tsai CH. Echocardiographic flow pattern of patent ductus arteriosus: a guide to indomethacin treatment in premature infants. Arch Dis Child Fetal Neonatal Ed 1999;81: F197 -200. 